\title{
Aspectos da distribuição de Culex (Culex) quinquefasciatus Say (Diptera, Culicidae) na região do rio Pinheiros, na cidade de São Paulo, Estado de São Paulo, Brasil
}

\author{
Sirlei Antunes de Morais ${ }^{1}$, Mauro Toledo Marrelli ${ }^{1} \&$ Delsio Natal $^{1}$
}

'Departamento de Epidemiologia da Faculdade de Saúde Pública, Universidade de São Paulo, Av. Dr. Arnaldo, 715, 01255 São Paulo-SP, Brasil. sirlei@usp.br

\begin{abstract}
Aspects of the distribution of Culex (Culex) quinquefasciatus Say (Diptera, Culicidae) in the region of the Pinheiros River, in the city of São Paulo, State of São Paulo, Brazil. The distribution of Culex (Culex) quinquefasciatus Say (1823) along of the margins of the Pinheiros river and the main factors that lead to the proliferation of the species were studied doing weekly collections of adult mosquitoes, in the period of one year, in three equidistant points in the margins of the river. For the collections of mosquitoes battery vacuum cleaner was used, for a period of five minutes. The mosquitoes were identified, differentiated according to the sex and counted. For verification of the physiological state, the females were differentiated in empty, with blood or eggs. A total of 35.684 mosquitoes were captured, all of them were identified as $C x$. quinquefasciatus, being $39.4 \%$ females and $60.6 \%$ males. The frequencies took different proportions between the points of collections and in a temporary series. The damaged environment of the Pinheiros river represents an excellent breeding site of $C x$. quinquefasciatus, confirmed by the occurrence of picks accentuated in the frequency of mosquitoes, with development in an explosive way and accumulation between the generations, after the rains and in summer times.
\end{abstract}

KEYWORDS. Culicidae; mosquitoes; population dynamic.

\begin{abstract}
RESUMO. Aspectos da distribuição de Culex (Culex) quinquefasciatus Say (Diptera, Culicidae) na região do rio Pinheiros, na cidade de São Paulo, Estado de São Paulo, Brasil. A distribuição de Culex (Culex) quinquefasciatus Say (1823) ao longo das margens do rio Pinheiros e os principais fatores que levam à proliferação da espécie foram estudados efetuando-se coletas semanais de mosquitos adultos, no período de um ano, em três pontos eqüidistantes às margens do rio. Para as coletas de mosquitos utilizou-se aspirador à bateria, por um período de cinco minutos. Os mosquitos foram identificados, diferenciados segundo o sexo e contados. Para verificação do estado fisiológico, as fêmeas foram separadas em vazias, com sangue e com ovos. Foram coletados 35.684 mosquitos, todos identificados como Cx. quinquefasciatus, sendo $39,4 \%$ fêmeas e $60,6 \%$ machos. As freqüências tomaram proporções diferentes entre os pontos de coletas e, em uma série temporal. O ambiente impactado do rio Pinheiros representa um excelente criadouro de Cx. quinquefasciatus, confirmado pela ocorrência de picos acentuados na freqüência de mosquitos, com desenvolvimento de forma explosiva e sobreposições entre as gerações, após as chuvas e em épocas de verão.
\end{abstract}

PALAVRAS-CHAVE. Culicidae; mosquitos; dinâmica populacional.

O rio Pinheiros pertence ao complexo hidráulico da Bacia de São Paulo, como afluente do rio Tietê. Com o desenvolvimento do Planalto Paulista no século XIX e a necessidade de fornecimento cada vez maior de energia elétrica, somado aos problemas de inundações da época, vários rios, dentre esses, o Tietê e o Pinheiros foram represados em vários pontos ao longo de seus trajetos (SP 2002). Com isso, esses ambientes lacustres tornaram-se paulatinamente lênticos e problemáticos.

Segundo o Boletim Técnico da Secretaria do Meio Ambiente, do Estado de São Paulo (SMA 2002), as águas do rio Pinheiros possuem níveis elevados de temperatura e sais minerais, como resultado da decomposição da matéria orgânica e industrial, constantemente despejada no seu leito. Como conseqüência, os dados de qualidade da água do rio apontam ausência de Oxigênio Dissolvido, o que as caracterizam como sendo anóxicas. A estagnação das águas do rio Pinheiros o torna ainda mais poluído e seu leito mais raso, permitindo a rápida proliferação de mosquitos adaptados a esse tipo de ambiente, como a espécie Culex (Culex) quinquefasciatus Say (1823).

Segundo Merrit et al. (1992), as larvas de $C x$. quinquefasciatus possuem o modo de alimentação do tipo coletor-filtrador (collecting-filtering). Essa via de alimentação apresenta um mecanismo de filtração bem desenvolvido, com adaptações principalmente nos músculos da faringe, tendo, após a ingestão, um seleto e pequeno conteúdo no estômago, mesmo com o meio externo sobrecarregado de substâncias alimentares (Clements 1999). Essa característica permite a sobrevivência da espécie em meios aquáticos poluídos. Tudo isso adicionado ao fato de que a alimentação dos imaturos de $C x$. quinquefasciatus se faz na coluna d'água, próxima à superfície do meio líquido, o que facilita a permanência destes em ambiente anóxico.

O mosquito $C x$. quinquefasciatus, comumente conhecido como pernilongo ou muriçoca, causa incômodo para os moradores próximos ao canal do rio Pinheiros. Essas 
ocorrências podem ser intensificadas, à medida que aumenta sua densidade e também pela elevada capacidade das fêmeas adultas em alimentar-se de sangue humano e de permanecer no ambiente de hematofagia (Forattini 2002).

Definiram-se como objetivos a distribuição de $C x$. quinquefasciatus ao longo das margens do rio Pinheiros e analisar os principais fatores que levam à proliferação da espécie. Para tanto, avaliou-se o ritmo de desenvolvimento da população de mosquitos, pela determinação da curva populacional e das frequiências por estado fisiológico das fêmeas e proporção entre os sexos, nos diferentes pontos da extensão do rio.

\section{MATERIALE MÉTODOS}

Este estudo foi realizado nas margens do rio Pinheiros, na cidade de São Paulo, Estado de São Paulo, mediante coleta de mosquitos adultos, no período de agosto de 2003 a agosto de 2004.

As informações mensais de temperatura atmosférica e precipitação pluviométrica na região foram adquiridas junto à Empresa Metropolitana de Águas e Energia S/A (EMAE/SP), na cidade de São Paulo, tendo uma estação de monitoramento junto ao canal do rio Pinheiros.

Para as atividades de monitoramento da população de mosquitos foram selecionados três pontos de amostragens, localizados ao longo da margem direita do rio Pinheiros. Os pontos foram especificados pelas letras A, B e C (Fig. 1).

O ponto A localiza-se na extremidade norte da extensão do rio Pinheiros, próximo a sua foz, no rio Tietê. Neste local, a margem do rio apresenta área de alagado, com vegetação

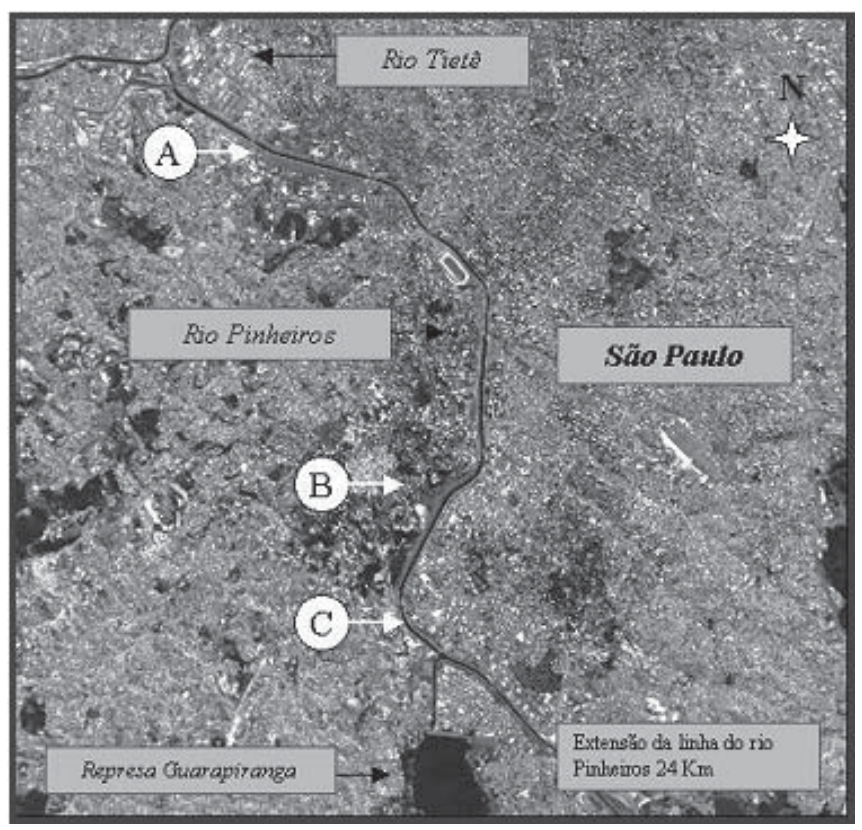

Fig. 1. Distribuição dos pontos de coleta de adultos de $C x$. quinquefasciatus, no rio Pinheiros, São Paulo, SP. Fonte: Imagem do satélite SPOT, 2002, cedido pela Intersat S/A. rasteira, do tipo gramíneo e algumas plantas aquáticas próximas ao leito do rio. Possui ainda, alguns arbustos próximos à estrada, paralelamente ao rio.

$\mathrm{A}$ área do ponto $\mathrm{B}$, próxima à metade da extensão do canal do rio, apresenta características semelhantes às do ponto $\mathrm{A}$.

$\mathrm{O}$ ponto de coleta $\mathrm{C}$ localiza-se na extremidade sul do canal do rio e fica próximo às represas Guarapiranga e Billings. Este ponto apresenta vegetação rasteira, do tipo gramíneo, sem sombreamento. Na outra margem, à esquerda do leito do rio, localiza-se as dependências do Projeto Pomar (projeto paisagístico desenvolvido pela Secretaria do Meio Ambiente do Governo do Estado, visando a recuperação do espaço geográfico às margens do rio) que constitui rica vegetação, com arbustos, árvores e diversidade de plantas e flores.

Em cada ponto foi instalada uma estaca, sendo que a região de borda explorada correspondeu um raio de $50 \mathrm{~m}$ desta marcação.

As coletas foram realizadas semanalmente, sendo utilizado aspirador à bateria do tipo adaptado por Natal \& Marucci (1984), o qual foi passado lentamente em meio à vegetação de borda, por um período de cinco minutos. Os mosquitos foram mortos imediatamente após a coleta e colocados em caixinhas entomológicas de plástico e embaladas em isopor com gelo reciclável.

Os mosquitos adultos foram identificados segundo Forattini (2002) e diferenciados segundo o sexo e contados.

Para verificação do estado fisiológico, as fêmeas foram diferenciadas em vazias, com sangue (com traços de sangue a cheias) e com ovos (sem traços de sangue).

$\mathrm{O}$ decurso da digestão do sangue ingerido pode ser acompanhado pela observação da aparência externa do abdome e, paralelamente, corresponderá às etapas do ciclo de desenvolvimento ovariano (Cella e Charlwood, citado por Forattini 2002).

Para o tratamento dos dados e avaliação das variações nas freqüências da população adulta de mosquitos foram adotadas as expressões abaixo relacionadas:

Frequiência de adultos $(F A)=$ Número total de mosquitos adultos por minuto de aspiração.

Número de Fêmeas vazias (FV); Número de Fêmeas com sangue (FS); Número de Fêmeas com ovos (FO).

Obs: Para FV, FS, e FO levou-se em conta o total coletado durante os cinco minutos de aspiração.

Para a análise estatística foram utilizados os softwares: SPSS $^{\circledR}$ 12.0 for Windows e SAS ${ }^{\circledR}$ 9.1.3 for Windows.

De acordo com teste de normalidade de KolmogorovSmirnov os dados das freqüências em todas as variáveis estudadas não seguem uma distribuição normal $(p<<0,00)$. Em vista disso, as comparações foram efetuadas através de testes Não-Paramétricos (Kruskal-Wallis) (Siegel 1975).

\section{RESULTADOS}

A distribuição da população de mosquitos. Durante o período de estudo foram coletados 35.684 mosquitos adultos da espécie $C x$. quinquefasciatus, nos três pontos de coleta 


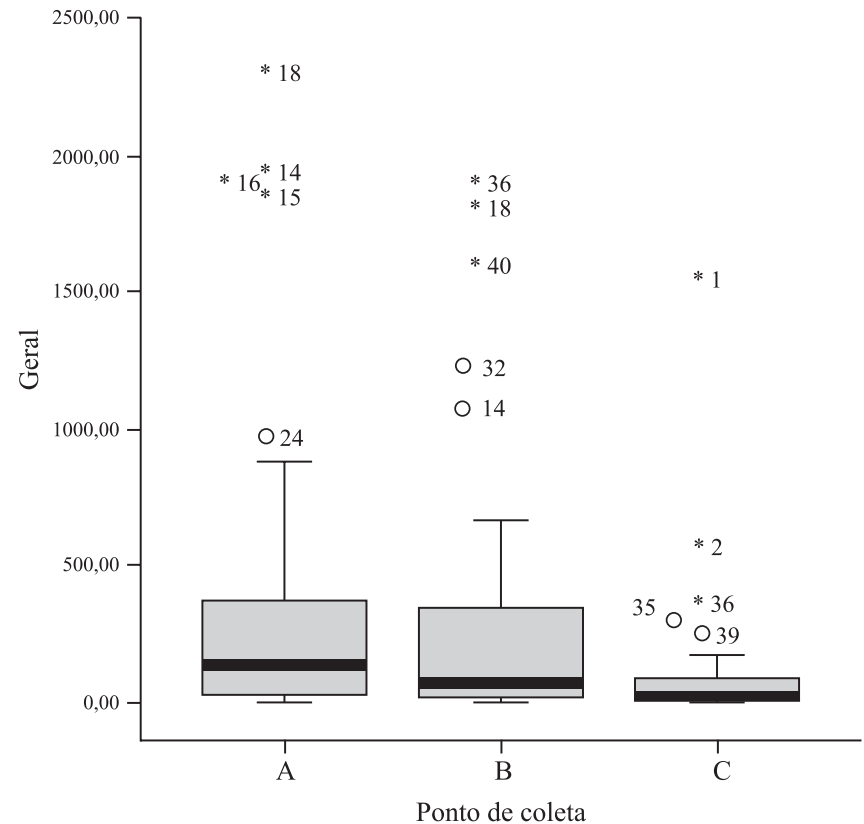

Fig. 2. Distribuição da população de Culex quinquefasciatus, segundo pontos de coleta, no rio Pinheiros, São Paulo, SP. Agosto de 2003 a agosto de 2004.

explorados. Esta espécie demonstrou exclusividade entre os culicídeos.

As médias das freqüências de adultos coletados apresentam diferenças significativas entre os pontos $(\mathrm{p}<0,05)$ (Fig. 2). O ponto A apresentou frequiência maior de mosquitos adultos, com um total de 17.079 indivíduos coletados e FA máxima de 474 mosquitos. Em seguida, o ponto B, com um total de 13.885 indivíduos coletados e FA máxima de 378 mosquitos. Por último, o ponto $\mathrm{C}$, com 4.720 exemplares de mosquitos adultos coletados. No mês de agosto este ponto
Tabela I. Distribuição das temperaturas médias máxima e mínima, com Coeficiente de Variação e Pluviosidade, segundo mês de coleta, na região do rio Pinheiros, São Paulo, SP. Agosto 2003 a julho de 2004.

\begin{tabular}{cccc}
\hline \multirow{2}{*}{ Mês } & Temp. Máx. & Temp. Mín. & Pluviosidade \\
\cline { 2 - 4 } & ${ }^{\circ} \mathrm{C}(\mathrm{CV})$ & ${ }^{\circ} \mathrm{C}(\mathrm{CV})$ & $\mathrm{mm}$ \\
\hline ago & $19,9(26,9)$ & $11,3(22,8)$ & 0,7 \\
set & $22,5(28,8)$ & $13,8(18,4)$ & 0,9 \\
out & $24,5(20,0)$ & $15,5(15,5)$ & 2,3 \\
nov & $25,5(18,4)$ & $16,8(15,7)$ & 6,5 \\
dez & $27,4(17,9)$ & $18,3(9,3)$ & 5,8 \\
jan & $24,9(14,3)$ & $18,0(9,1)$ & 9,8 \\
fev & $25,5(15,2)$ & $18,0(8,5)$ & 12,5 \\
mar & $25,3(12,3)$ & $17,8(11,4)$ & 7,5 \\
abr & $25,6(11,5)$ & $18,2(11,0)$ & 13,8 \\
mai & $20,7(16,9)$ & $13,8(20,7)$ & 6,9 \\
jun & $21,3(17,5)$ & $12,3(19,4)$ & 9,5 \\
jul & $19,6(23,3)$ & $12,2(22,1)$ & 9,2 \\
\hline
\end{tabular}

Fonte: EMAE/ SP / 2004

apresentou FA máxima de 321 mosquitos, sendo que no restante do ano, manteve um patamar de 0 a 73 mosquitos coletados por minuto de aspiração.

Na Fig. 2 podem ser observados valores extremos aos normais (outlies). Os outlies representam as coletas efetuadas em períodos de explosão de mosquitos e serão discutidos adiante.

As freqüências de mosquitos machos e fêmeas apresentam diferenças significantes entre os três pontos de coleta $(\mathrm{p}<$ $0,05)$. De modo geral, as fêmeas de $C x$. quinquefasciatus estiveram distribuídas em menor número nos abrigos, comparando-se com a freqüência de machos adultos (Tab. II).

As populações de fêmeas vazias apresentam diferenças significativas entre os três pontos de coleta $(p<0,05)$. Não existem diferenças entre as frequiências de fêmeas com ovos e com sangue $(\mathrm{p}>0,05)$.

Tabela II. Distribuição mensal das freqüências (\%) de adultos de Cx. quinquefasciatus, segundo sexo e estado fisiológico das fêmeas, no rio Pinheiros, São Paulo, SP. Agosto de 2003 a agosto de 2004.

\begin{tabular}{|c|c|c|c|c|c|c|}
\hline \multirow{3}{*}{ Mês } & \multicolumn{3}{|c|}{ Fêmeas } & \multirow{2}{*}{ Fêmeas } & \multirow{2}{*}{ Machos } & \multirow{3}{*}{ Total } \\
\hline & $\mathrm{FV}^{*}$ & FS* & FO* & & & \\
\hline & $\mathrm{N}(\%)$ & $\mathrm{N}(\%)$ & $\mathrm{N}(\%)$ & $\mathrm{N}(\%)$ & $\mathrm{N}(\%)$ & \\
\hline ago & $1097(84,4)$ & $47(3,6)$ & $155(11,9)$ & $1299(41,3)$ & $1845(58,7)$ & 3144 \\
\hline set & $288(72,5)$ & $13(3,3)$ & $96(24,2)$ & $397(44,4)$ & $498(55,6)$ & 895 \\
\hline out & $152(80,0)$ & $1(0,5)$ & $37(19,5)$ & $190(30,1)$ & $442(69,9)$ & 632 \\
\hline nov & $1508(92,7)$ & $7(0,4)$ & $112(6,9)$ & $1627(32,9)$ & $3325(67,1)$ & 4952 \\
\hline $\operatorname{dez}$ & $2227(87,3)$ & $1(0,0)$ & $324(12,7)$ & $2552(45,6)$ & $3041(54,4)$ & 5593 \\
\hline jan & $1963(94,6)$ & $18(0,9)$ & $93(4,5)$ & $2074(38,4)$ & $3327(61,6)$ & 5401 \\
\hline fev & $688(91,9)$ & $9(1,1)$ & $88(11,2)$ & $785(37,6)$ & $1300(62,4)$ & 2085 \\
\hline mar & $396(91,9)$ & $3(0,7)$ & $32(7,4)$ & $431(32,3)$ & $904(67,7)$ & 1335 \\
\hline abr & $1234(84,3)$ & $26(1,8)$ & $204(13,9)$ & $1464(44,8)$ & $1806(55,2)$ & 3270 \\
\hline mai & $1266(81,7)$ & $38(2,5)$ & $245(15,8)$ & $1549(34,8)$ & $2897(65,2)$ & 4446 \\
\hline jun & $1010(88,5)$ & $31(2,7)$ & $100(8,8)$ & $1141(45,5)$ & $1366(54,5)$ & 2507 \\
\hline jul & $288(79,1)$ & $5(1,4)$ & $71(19,5)$ & $364(46,0)$ & $427(54,0)$ & 791 \\
\hline ago & $140(68,6)$ & $23(11,3)$ & $41(20,1)$ & $204(32,2)$ & $429(67,8)$ & 633 \\
\hline Total & $12257(87,1)$ & $222(1,6)$ & $1598(11,4)$ & $14077(39,4)$ & $21607(60,6)$ & 35684 \\
\hline
\end{tabular}

* FV: Fêmeas vazias; FS: Fêmeas com sangue; FO: Fêmeas com ovos. 


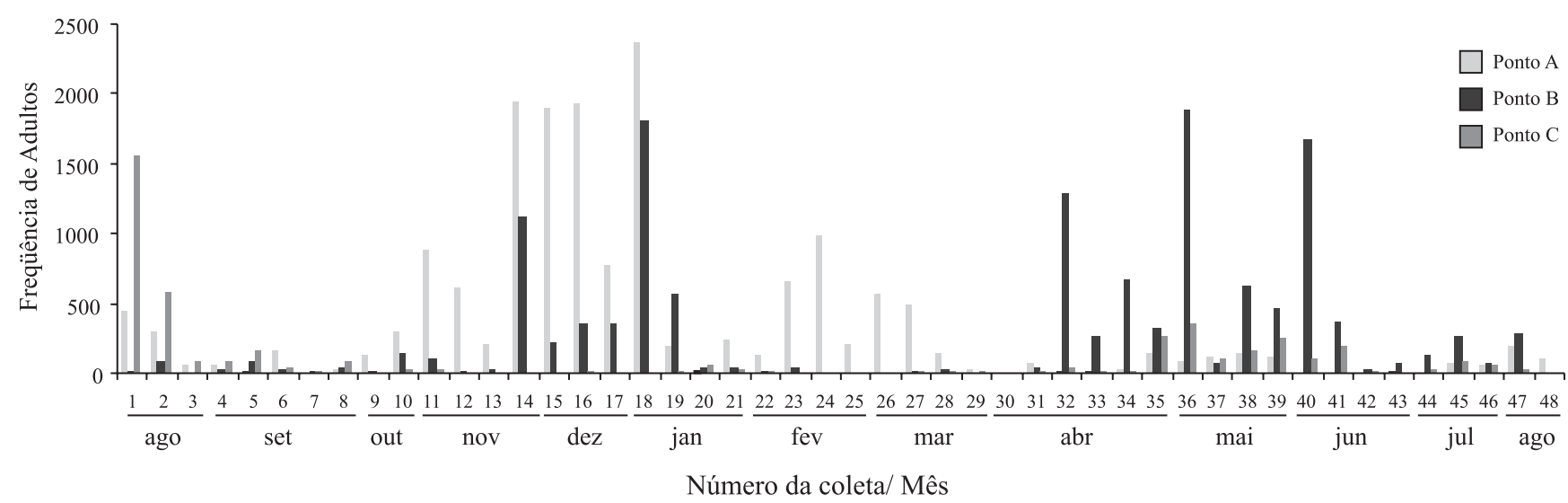

Fig. 3. Distribuição de mosquitos adultos de Cx. quinquefasciatus, segundo mês e número da coleta, em três pontos de monitoramento às margens do rio Pinheiros, SP. Agosto de 2003 a agosto de 2004.

As freqüências dentro da curva populacional. Para avaliar as diferenças na flutuação de $C x$. quinquefasciatus no rio Pinheiros, tomemos como exemplo a curva populacional formada pelas frequiências do ponto $\mathrm{A}$, durante as coletas $9 \mathrm{a}$ 13, entre os meses de outubro e novembro de 2004 (Fig. 3).

A curva acima descrita refere-se ao desenvolvimento normal de uma geração de mosquitos. A coleta de número 9 apresentou proporção maior de fêmeas com ovos do que nos outros períodos (FV 39\%; FS 4\% e FO 57\%) e reduzida proporção de fêmeas ( somou maior proporção de fêmeas vazias (FV 96\%; FS 0\% e FO 4\%) e menor número de fêmeas para machos (ㅇ 27\% e o $73 \%$ ). Na coleta de número 11 houve $100 \%$ de fêmeas vazias, tendo poucas fêmeas nos abrigos com relação ao número de machos ( $\$ 28 \%$ e o $72 \%$ ). A coleta de número 12 apresentou um pequeno percentual de fêmeas com sangue e com ovos (FV 88\%; FS 3\% e FO 9\%), sendo 30\% Fêmeas e 70\% machos. Já na coleta de número 13 houve grande quantidade de fêmeas com ovos (FV 64\%; FS 0\% e FO 36\%) e menor número de fêmeas com relação ao número de machos (+ $24 \%$ e o $0^{7} 76 \%$ ), com uma queda na freqüência geral da população (FA 15), fechando assim um ciclo na geração de mosquitos.

As freqüências dentro das séries de explosões. No caso dos picos isolados (Fig. 3), foram observados valores diferentes entre a taxa de sexo e estado fisiológico dos mosquitos adultos. Na coleta 15 , por exemplo, realizada no início do mês de dezembro, além de um aumento na freqüência geral de mosquitos (FA 381), houve aproximação na proporção de fêmeas para machos ( + + $49 \%$ e o $^{\star} 51 \%$ ). Ocorreu ainda, grande quantidade de fêmeas vazias e números razoáveis de fêmeas com ovos (FV 81\%; FS 0\% e FO 19\%).

\section{DISCUSSÃO}

A distribuição da população de mosquitos. As condições adequadas para o desenvolvimento de imaturos e também de adultos nos meses de janeiro a março fizeram com que houvesse um desenvolvimento populacional somente no ponto A, com o crescimento de uma geração inteira de mosquitos (coletas 22 a 29) (Fig. 3). Durante o período de maior precipitação (Tab. I), a água parece ter arrastado boa parte das formas imaturas de mosquitos para o lado norte do rio, nas proximidades do ponto A. Nesta extremidade do rio (Norte) existe maior concentração de matéria orgânica e mineral (SMA 2002), o que elucida para uma certa tendência da água a levar tanto os poluentes, como os ovos e as larvas dos mosquitos nessa mesma direção em épocas de alta pluviosidade.

No ponto $\mathrm{C}$ ocorreu um pico acentuado no mês de agosto e, no restante do ano, a freqüência de $C x$. quinquefasciatus se manteve em baixos níveis e de forma contínua. Acerca deste pico (coletas 2 e 3, Fig. 3), pode ser observado nos dados de campo, uma quantidade muito superior de fêmeas neste intervalo, o que denota um aumento nas atividades reprodutivas, dentre essas, a procura por criadouros. De modo geral, a proporção de fêmeas neste ponto de coleta esteve próxima a quantidade de machos, algumas vezes até ultrapassando esta. Com isto, pode ser inferido que as condições da água e do ambiente nesta extremidade do rio (Sul) são mais favoráveis para as atividades reprodutivas da população de $C x$. quinquefasciatus.

Na extremidade sul do rio Pinheiros, a temperatura da água é sempre maior com relação às outras áreas, devido ao fenômeno da poluição térmica, variando entre $17 \mathrm{a} 31^{\circ} \mathrm{C}$ mesmo em épocas de inverno (SMA 2002), exceto em dias de chuva. $\mathrm{Na}$ ocasião supramencionada, ocorreram baixos índices de pluviosidade, conforme demonstrado na Tab. I. Esse fato, adicionado às temperaturas ótimas da água em épocas de frio, pode ter favorecido a eclosão de forma explosiva da população de imaturos nessa região. Em vista disso, este local pode ser considerado como área de risco para explosões de $C x$. quinquefasciatus, mesmo em períodos de inverno. Adicionado a isso, deve ser levado em consideração as condições da vegetação na margem esquerda do rio, onde se localiza o Projeto Pomar. A presença de plantas com flores aumenta indiretamente as atividades reprodutivas das fêmeas, pela maior disponibilidade de energia. 
Foram realizadas, no ponto $\mathrm{C}$, limpezas contínuas na vegetação marginal, a partir do mês de agosto. Esse procedimento pode ter propiciado o deslocamento dos mosquitos adultos para outros abrigos mais favoráveis, em outros pontos da margem do rio, que explica a pouca freqüência de mosquitos coletados durante o ano de estudo neste ponto de monitoramento.

O ritmo de desenvolvimento dentro da curva populacional. $\mathrm{Na}$ estação quente ocorre sobreposição de gerações, principalmente pelo ritmo contínuo nas atividades de reprodução das fêmeas adultas de $C x$. quinquefasciatus. E, no período com temperaturas mais baixas, o crescimento dos indivíduos se faz de forma simultânea (Fig. 3). Em geral, a população teve um desenvolvimento mais acelerado em épocas de calor e após o período de chuvas.

Em países tropicais, como no caso do Brasil, os níveis de variação da temperatura geralmente têm característica linear, principalmente nos Estados próximos à linha do equador, como o Estado de Pernambuco, onde a temperatura, em 1990, variou entre 24 a $28^{\circ} \mathrm{C}$ e umidade relativa entre 67 a $85 \%$ (Regis 1995). No caso da cidade de São Paulo, que fica mais próxima dos trópicos, a temperatura esteve entre 12 a $27^{\circ} \mathrm{C}$, com coeficiente de variação mensal de até $28 \%$, no período de estudo (Tab. I), o que implica em ausência de grandes extremos, comparandose com países temperados.

Segundo Harrington \& Stork (1995), o período de chuva tem influência direta e indireta na flutuação da população de mosquitos. A água da chuva, além de atrapalhar o vôo dos adultos e conseqüentemente à atividade destes, faz uma remoção de larvas e ovos ao longo da extensão da área. Em conseqüência, após o período de chuva, a umidade relativa do ar aumenta.

Com uma boa disponibilidade de vapor d'água no ambiente e temperatura adequada, as funções dos organismos ganham condições favoráveis para se desenvolver, podendo produzir um ritmo de crescimento mais acelerado na população de insetos, como mostrado na Fig. 3. Durante os meses de outubro, fevereiro e abril, houve a ocorrência de chuvas mais intensas, com um período de queda desta, em concomitância o inverso ocorreu na população de mosquitos; ou seja, redução na frequiência, seguida de aumento da população.

Outro fator para a queda da frequiência de adultos na curva populacional é o envelhecimento natural da população (Clements 1999). A Tab. II mostra a evolução do período de vida dos mosquitos, observando-se os meses mais frios, onde parece não haver sobreposição de gerações. As fêmeas aparecem nos meses de agosto e setembro/03 com uma proporção maior de fêmeas com sangue (idade fértil) nos abrigos explorados, seguido de redução no número de mosquitos. Nos períodos subseqüentes ocorre menor proporção de fêmeas com sangue, com aumento na frequiência de fêmeas vazias e no número de mosquitos, o que leva a inferir o envelhecimento da população anterior.

$\mathrm{Na}$ população com desenvolvimento nos períodos mais quentes, a condição acima mencionada não é tão evidente. Segundo Clements (1999), a primeira geração após o período de inverno, possui menos reserva de energia na camada de gordura do corpo, como herança da geração passada, o que influencia diretamente no processo de fertilização. Como contra-balanço a primeira geração (nos períodos mais quentes) se desenvolve mais intensamente e de uma maneira contínua, pelo imediatismo das condições favoráveis do clima.

O ritmo de desenvolvimento em série de explosões. $\mathrm{Na}$ Fig. 2 foram observados valores extremos aos normais (outlies) nos três pontos de coleta. Nestas ocasiões foram coletados os valores máximos de Freqüência de Adultos (FA). Além disso, foi constatado que nesses períodos a freqüência de fêmeas vazias é maior do que em uma curva populacional normal. E ainda, a proporção entre machos e fêmeas é aproximada. Essas características demonstram que existe maior quantidade de fêmeas jovens no campo, como resultado de emergências recentes e em massa na população de mosquitos. As explosões são características marcantes do rio Pinheiros, formando sobreposições na curva populacional. Além disso, implicam em diferenças na dinâmica da população de $C x$. quinquefasciatus, dentro de uma linha de normalidade.

Ressalta-se que neste ecótopo são realizadas aplicações de controle na população de mosquitos, sob responsabilidade do órgão público do município de São Paulo. Essas ações motivaram para uma análise mais criteriosa do impacto produzido nas freqüências da população de $C x$. quinquefasciatus, que será discutida em um trabalho posterior a este.

\section{CONCLUSÕES}

A falta de fluxo e as características peculiares da água fazem do rio Pinheiros um excelente criadouro de $C x$. quinquefasciatus.

O estudo mostra a existência de uma variedade de gerações de $C x$. quinquefasciatus ao longo do ano, com períodos de explosão da população, sendo que na estação quente ocorre sobreposição das gerações e nos períodos com temperaturas mais baixas, o número de mosquitos adultos diminui acentuadamente, formando somente uma curva populacional, pelo crescimento simultâneo dos mosquitos.

Os dados indicam maior concentração de mosquitos no sentido norte, próximo ao rio Tietê. Contudo, no ponto de monitoramento ao sul da extensão do rio, as fêmeas apresentam maior atividade reprodutiva.

As frequiências no estado fisiológico e na taxa de sexo dos mosquitos apresentam diferentes proporções em uma curva populacional e em séries de explosões, em toda a extensão do rio.

\section{REFERÊNCIAS}

Clements, A. N. 1999. The biology of mosquitoes: sensory reception and behaviour. Wallingford, CABI, v.2., 740 p. Forattini, O. P. 2002. Culicidologia Médica: identificação, 
biologia e epidemiologia. São Paulo, EDUSP, v.2., 860 p.

Harrington, R \& N. E. Stork. 1995. Insect in a changing environment. London, Academic Press, 535 p.

Merritt, R. W; R. H Dadd; E. D. Walker. 1992. Walker. Feeding behavior, natural food, and nutritional relationships of larval mosquitoes. Annual Review Entomology 37: 349-76.

Natal, D. \& D. Marucci. 1984. Aparelho de sucção tipo aspirador para captura de mosquitos. Revista Saúde Publica 18: 418-20.

Regis, L; M. H. N. L. Silva-Filha; C. M. F. Oliveira; E. M. Rios; S. B. Silva \& A. Furtado. 1995. Integrated control measures against
Culex quinquefasciatus, the vector of filariasis in Recife. Memórias do Instituto Oswaldo Cruz 90: 115-19.

Siegel, S. 1975. Estatística não-paramétrica para as ciências do comportamento. São Paulo, McGraw-Hill do Brasil, 350 p.

[SMA] São Paulo. Secretaria do Estado do Meio Ambiente. Coordenadoria de Planejamento Ambiental. 2002. Informações básicas para o planejamento ambiental. São Paulo, SMA, 84p.

[SP] São Paulo (Estado). Secretaria do Meio Ambiente e Governo do Estado de São Paulo. 2002. O Rio Pinheiros. São Paulo, Centro de Editoração da Secretaria do Meio Ambiente de Estado do São Paulo. $96 \mathrm{p}$.

Recebido em 16/03/2006; aceito em 10/07/2006 